\title{
Factors affecting long term results of successfully treated amblyopia: initial visual acuity and type of amblyopia
}

\author{
Shmuel Levartovsky, Moshe Oliver, Nava Gottesman, Michael Shimshoni
}

\begin{abstract}
Aims-The study aimed to assess the effect of initial visual acuity and type of amblyopia on the long term results of successfully treated amblyopia.

Methods-The visual acuity of 94 patients, who had been successfully treated for unilateral amblyopia by occlusion of the good eye and followed up to the age of 9 years, was examined 6.4 years, on average, after cessation of treatment. Patients were divided into two groups according to the depth of amblyopia before occlusion therapy was started: those with visual acuity between $20 / 60$ and $20 / 100$ and those with visual acuity of $20 / 100$ or worse.
\end{abstract}

Results-Deterioration of visual acuity was observed in $42 \%$ of patients in the first group and in $63 \%$ of patients in the second group. Their average deterioration, as measured by the Snellen chart, was 0.58 and 1.54 lines, respectively. The results were also assessed by the division of patients into three groups according to the type of amblyopia: strabismic, strabismic anisometropic, and anisometropic. Deterioration of visual acuity occurred in $46 \%, 79 \%$, and $36 \%$ of patients in these three groups, with an average deterioration on the Snellen chart of $0 \cdot 70,2 \cdot 04$, and 0.64 lines, respectively.

Conclusion-It is concluded that low initial visual acuity and strabismic anisometropic amblyopia are risk factors for deterioration of visual acuity in the long term, following the successful earlier treatment of eyes with amblyopia.

Department of

Ophthalmology,

Kaplan Hospital,

Rehovot, affiliated

with The Hebrew

University-Hadassah

Medical School,

Jerusalem

S Levartovsky

M Oliver

N Gottesman

Department of Applied Mathematics and

Computer Science, The Weizmann

Institute of Science,

Rehovot, Israel

M Shimshoni

Correspondence to: Moshe Oliver, MD,

Department of

Ophthalmology, Kaplan

Hospital, Rehovot 76100,

Israel.

Accepted for publication 16 November 1994
(Br f Ophthalmol 1995; 79: 225-228)

Occlusion of the good eye is the most widely accepted and probably the most effective method of treatment for amblyopia. After the treatment ends, however, the improved visual acuity in the amblyopic eye tends to deteriorate. In an earlier study, ${ }^{1}$ we showed that visual deterioration had occurred in $55 \%$ of successfully treated eyes when measured at the long term follow up examination. We also found that the age at which treatment was started did not significantly affect the mean extent of deterioration or the mean visual acuity in the long term. In the present study we considered two additional variables as possible risk factors and examined their long term effects on the deterioration of visual acuity in eyes that had earlier been successfully treated for amblyopia: the initial depth of amblyopia - that is, the visual acuity of the amblyopic eye before the occlusive treatment was started, and the type of amblyopia.

\section{Patients and methods}

Between 1974 and 1979, 350 children with unilateral amblyopia were diagnosed and treated at the outpatient eye clinic of Kaplan Hospital, Rehovot. Our earlier report on that study ${ }^{1}$ contains a detailed description of the patients and methods, which is also applicable to the present work. Treatment consisted only of occlusion of the good eye. In each case, after the best visual acuity in the amblyopic eye was attained, we continued to monitor vision at intervals of at least every 3 months for a year. Thereafter, visual acuity was checked during the course of a complete eye examination performed at least once a year until the child was 9 years old. Whenever necessary - that is, if visual acuity had deteriorated, occlusion of the good eye was reintroduced for as long is it took to restore vision to the level previously attained.

Inclusion of a patient in the present long term follow up study was contingent on the following criteria being met at the time that amblyopia was diagnosed and during the subsequent period of treatment and monitoring.

(1) Compliance of the child with an age suitable Snellen chart for the initial visual acuity examination. As an exception to this criterion there were 12 children with unilateral strabismus who were too young (under 3 years old) at the beginning of the study to be tested in this way. We estimated the visual acuity of the amblyopic eye in these children by determining the density of the filter required to induce a shift of fixation to the squinting or presumed amblyopic eye.

(2) An initial visual acuity of $20 / 60$ or worse in the amblyopic eye.

(3) Full compliance with the instructions of the medical staff during the period of therapy and follow up.

(4) Cessation of occlusion therapy at least 1 year before the present study.

On the basis of these criteria, 157 children were eligible for inclusion in this study. Of these, $104(66 \%)$ responded to our invitation and presented themselves for the long term follow up examination. Ninety four children who were monitored for visual acuity up to at least the age of 9 years were included in the study.

At the long term follow up examination, the best corrected visual acuity on the Snellen 
Table 1 Number (\%) of children in each initial visual acuity group showing deterioration in visual acuity at the long term follow up examination

\begin{tabular}{lll}
\hline $\begin{array}{l}\text { Initial visual } \\
\text { acuity group }\end{array}$ & $\begin{array}{l}\text { Number of } \\
\text { children }\end{array}$ & $\begin{array}{l}\text { Number of children with } \\
\text { visual acuity deterioration }\end{array}$ \\
\hline $20 / 60-20 / 100$ & 45 & $19(42)$ \\
$20 / 100$ or less & 49 & $31(63)$ \\
Total & 94 & $50(53)$ \\
\hline
\end{tabular}

chart after refraction was recorded. Cycloplegic refraction, when necessary, was performed 1 hour after administration of cyclopentolate $1 \%$, twice over a 10 minute interval, followed by subjective refraction 1 week later. Orthoptic and fundal examinations were also performed.

In order to evaluate the influence of initial visual acuity (the visual acuity before any treatment) or the type of amblyopia on the long term results, the following variables were analysed.

\section{INITIAL VISUAL ACUITY}

Two groups of amblyopic eyes were defined. The initial visual acuity was between $20 / 60$ and $20 / 100$ in the first group (45 patients), $20 / 200$ or worse in the second (49 patients).

\section{TYPE OF AMBLYOPIA}

Three groups were defined: strabismic amblyopia, where a cover test at the initial examination had demonstrated strabismus of the amblyopic eye, and the spherical equivalent difference between the eyes was less than 1.00 dioptre (56 patients); strabismic anisometropic amblyopia, where a cover test at the initial examination had demonstrated strabismus of the amblyopic eye, and the spherical equivalent difference between the eyes was 1.00 dioptre or more (24 patients); anisometropic amblyopia, where a cover test at the initial examination had demonstrated no strabismus and the spherical equivalent difference between the eyes was 1.00 dioptre or more (14 patients).

\section{Data analysis and calculation of visual acuity deterioration}

Each line on the Snellen chart was assigned a score, as follows: $<20 / 200,1 ; 20 / 200,2$; 20/100, 3; 20/80, 4; 20/70, 5; 20/60, 6; 20/50, $7 ; 20 / 40,8 ; 20 / 30,9 ; 20 / 25,10 ; 20 / 20,11$. We used this Snellen score rather than the $\log$ MAR score; we did so because throughout the study the visual acuity of the patients was determined by using Snellen charts rather than $\log$ MAR ones.

The visual acuity score at the long term follow up examination was compared with that attained by the child upon termination of occlusive therapy. The difference between them was the 'deterioration score', which reflects the amount of deterioration in visual acuity. For each of the groups defined above, the average amount of deterioration was calculated by dividing the sum of the deterioration scores by the number of children in the group.

The data for each child, including visual acuity before occlusion was started, refraction of both eyes and cover test findings at the commencement of treatment, best visual acuity at termination of treatment, and best visual acuity at the long term follow up examination, were prepared for computer analysis. The Student's $t$ test and the $\chi^{2}$ test were used for statistical evaluation. Results were assumed to be significant if $p<0.05$.

\section{Results}

Of the 94 patients who presented themselves for the long term follow up examination, 45 were male and 49 were female. Their ages at the time of the examination ranged from 9.0 to 20.0 years (mean 13.8 (SD 2.4 ) years). The examination was performed between $1 \cdot 1$ and 9.8 years (average $6.4(1.8)$ years) after discontinuation of occlusive treatment. Most of the children ( $84 \%$ ) were examined between 4 and 9 years after the treatment was discontinued.

Forty four $(47 \%)$ of the examinees were found to have maintained the visual acuity achieved when occlusion treatment was terminated, while in $50(53 \%)$ the visual acuity had deteriorated.

\section{INITIAL VISUAL ACUITY AND LONG TERM} VISUAL ACUITY DETERIORATION

In both groups, some of the patients showed deterioration of visual acuity at the long term follow up examination. As shown in Table 1, of the 45 patients with initial visual acuity between 20/60 and 20/100, $19(42 \%)$ showed visual deterioration. Of the 49 patients with an initial visual acuity of $20 / 100$ or worse, 31 (63\%) showed visual deterioration. This difference between the findings in the two groups is statistically significant.

Table 2 presents the mean visual acuity scores at the beginning and end of occlusive treatment and at the long term follow up examination, and the mean deterioration scores of the two groups. Obviously the mean visual acuity was initially much higher in the 20/60-20/100 group, the mean visual acuity scores of the two groups at the end of the treatment were not statistically different. At the long term follow up examination, however, the mean deterioration score as measured on the Snellen chart was only 0.58 lines in the

Table 2 Mean visual acuity score (SE) for each initial visual acuity group at the beginning and end of occlusive treatment and at the long term follow up examination, and mean deterioration scores

\begin{tabular}{lllll}
\hline Initial visual acuity & $\begin{array}{l}\text { Mean visual acuity score } \\
\text { before the start of treatment }\end{array}$ & $\begin{array}{l}\text { Mean visual acuity score } \\
\text { at the end of treatment }\end{array}$ & $\begin{array}{l}\text { Mean visual acuity score at the } \\
\text { long term follow up examination }\end{array}$ & $\begin{array}{l}\text { Mean deterioration } \\
\text { score }\end{array}$ \\
\hline $20 / 60-20 / 100(\mathrm{n}=45)$ & $4.51(0 \cdot 17)$ & $8 \cdot 33(0 \cdot 16)$ & $7 \cdot 76(0.28)$ & $0.58(0 \cdot 25)$ \\
$20 / 100$ or less $(\mathrm{n}=49)$ & $1.45(0.07)$ & $8 \cdot 20(0 \cdot 19)$ & $6 \cdot 76(0.32)$ & $1 \cdot 45(0.30)$ \\
\hline
\end{tabular}


Table 3 Number of children in each type of amblyopia group showing deterioration in visual acuity at the long term follow up examination

\begin{tabular}{ll}
\hline $\begin{array}{l}\text { Type of amblyopia } \\
\text { No of children) }\end{array}$ & $\begin{array}{l}\text { No of children (\%) } \\
\text { with visual deterioration }\end{array}$ \\
\hline Strabismic amblyopia $(\mathrm{n}=56)$ & $26(46)$ \\
$\begin{array}{l}\text { Strabismic and anisometropic } \\
\text { amblyopia }(\mathrm{n}=24)\end{array}$ & $19(79)$ \\
Anisometropic amblyopia $(\mathrm{n}=14)$ & $5(36)$ \\
Total (n=94) & $50(53)$ \\
\hline
\end{tabular}

20/60-20/100 group compared with 1.45 lines in the other group. Thus, the amount of deterioration was significantly higher in children who had started treatment with a visual acuity of $20 / 100$ or worse in the amblyopic eye.

TYPE OF AMBLYOPIA AND LONG TERM VISUAL ACUITY DETERIORATION

Deterioration in visual acuity was seen in all three groups at the long term follow up examination (Table 3). Of the 56 patients in the strabismic amblyopic group, 26 (46\%) showed deterioration in vision from that attained at termination of treatment. Of the 24 patients in the strabismic anisometropic amblyopia group, 19 (79\%) patients showed deterioration. Of the 14 patients in the anisometropic amblyopia group, five (36\%) showed deterioration. The percentage of patients showing deterioration in visual acuity was significantly higher in the strabismic anisometropic amblyopia group than in the other two groups $(p<0.01)$.

Table 4 presents the mean visual acuity scores of the three groups at the beginning and end of treatment and at the long term follow up examination. The mean visual acuity scores measured on each of these occasions did not differ significantly among the three groups. However, the amount of deterioration at the long term follow up examination was significantly higher in the strabismic anisometropic group than in the strabismic group $(p<0.01)$. Although the anisometropic group also showed less deterioration than the strabismic anisometropic group, the results are not statistically significant because of the small sample size.

\section{Discussion}

In our previous report, ${ }^{1}$ which included all of the patients in the present study, the long term results of occlusive treatment for amblyopia were analysed 6.4 years, on average, after the treatment was discontinued. We found that the visual acuity attained at the end of occlusive therapy was maintained in $45 \%$ of the successfully treated amblyopic eyes. This finding is in accordance with the results of other authors, ${ }^{2-8}$ who reported that $10-44 \%$ of treated eyes maintain their visual acuity at examinations performed 1-10 years after cessation of occlusive therapy. Our success rate was high because we included in our study only those children who had shown full compliance with the instructions of the medical staff during the period of therapy and the follow up.

In the previous study ${ }^{1}$ we also found that the age at which therapy was initiated did not significantly affect the visual acuity results or the extent of deterioration of visual acuity measured at the long term follow up examination.

In the present study we analysed the influence of two additional variables, initial visual acuity and type of amblyopia, on the extent of long term deterioration of visual acuity in successfully treated eyes. We found that in children with an initial visual acuity of $20 / 100$ or worse, the mean visual acuity achieved at the end of occlusive therapy was virtually identical with that of children with an initial visual acuity between $20 / 60$ and 20/100. Thus, the depth of amblyopia before the start of occlusive therapy had little effect on the visual acuity results at the end of treatment. Thereafter, however, more children whose initial visual acuity was $20 / 100$ or worse tended to deteriorate and the mean amount of deterioration in that group was greater than in children whose initial visual acuity was 20/60-20/100.

Ching and colleagues ${ }^{9}$ studied 116 strabismic patients with amblyopia treated by occlusion of the preferred eye. In $48 \%$ of their patients, amblyopia did not recur after cessation of occlusive therapy, while the rest required reinstitution of part time occlusion therapy because the amblyopia had recurred. The mean initial visual acuity before the start of treatment was found to have been significantly lower in the group that needed further treatment. Although the influence of the initial visual acuity on the long term visual acuity results was not evaluated in that study, the authors suggest that the more profoundly amblyopic patients should be followed more closely after therapy is discontinued, since they are at greater risk of developing recurrent amblyopia. In the present study we showed that in patients with deep amblyopia at the beginning of occlusion therapy, the visual acuity can be expected to deteriorate even years after discontinuation of therapy.

In order to evaluate the influence of different types of amblyopia on the long term visual acuity results, we defined three groups of

Table 4 Mean visual acuity score (SE) for each type of amblyopia at the beginning and end of occlusive treatment and at the long term follow up examination, and mean deterioration scores

\begin{tabular}{lllll}
\hline $\begin{array}{l}\text { Type of amblyopia } \\
\text { (No of children) }\end{array}$ & $\begin{array}{l}\text { Mean visual acuity score } \\
\text { before the start of treatment }\end{array}$ & $\begin{array}{l}\text { Mean visual acuity score } \\
\text { at the end of treatment }\end{array}$ & $\begin{array}{l}\text { Mean visual acuity score at the } \\
\text { long term follow up examination }\end{array}$ & $\begin{array}{l}\text { Mean deterioration } \\
\text { score }\end{array}$ \\
\hline $\begin{array}{l}\text { Strabismic amblyopia } \\
(\mathrm{n}=56)\end{array}$ & $2.91(0.23)$ & $8.13(0.18)$ & $7.43(0.28)$ & $0.70(0.22)$ \\
$\begin{array}{c}\text { Strabismic-anisometropic } \\
\text { amblyopia (n=24) }\end{array}$ & $2.83(0.37)$ & $8.71(0.13)$ & $6.67(0.50)$ & $2.04(0.46)$ \\
$\begin{array}{c}\text { Anisometropic amblyopia } \\
(\mathrm{n}=14)\end{array}$ & $3.07(0.53)$ & $8.07(0.37)$ & $7.43(0.49)$ & $0.64(0.51)$ \\
\hline
\end{tabular}


patients according to the type of amblyopia that is, strabismic, strabismic anisometropic, and anisometropic. We found a greater tendency towards deterioration of visual acuity in the strabismic anisometropic group; the mean deterioration in that group was about three times higher than in the other two groups.

In a study of 89 patients treated for strabismic amblyopia and followed to at least 9 years of age, Scott and Flabetich Dickey ${ }^{10}$ reported deterioration of visual acuity in $25 \%$ of the patients by the time they reached a mean age of 16 years. In our series, deterioration of visual acuity at the long term follow up examination was found in $46 \%$ of the patients with strabismic amblyopia. The difference between the two sets of results might be explained by the fact that in the former series only $19 \%$ of the patients had pretreatment visual acuity of $20 / 100$ or worse, compared with $52 \%$ of the patients in our series. As mentioned above, patients with this low level of pretreatment visual acuity tend to show greater deterioration in visual acuity at the long term follow up examination.

Kutschke et al described visual acuity results in 124 children with anisometropic amblyopia. ${ }^{11}$ Only 66 patients had completed occlusion therapy; of these, $16(24 \%)$ subsequently regressed by two or more Snellen chart lines. The length of the follow up and the number of patients who regressed by only one line are not recorded. In our study, 14 children with anisometropic amblyopia were evaluated. Of these, five (36\%) showed deterioration of visual acuity at the long term follow up examination. However, only three of these five children $(21 \%)$ showed a deterioration of two or more Snellen chart lines.

Oster et al ${ }^{12}$ described a series in which 182 patients with amblyopia due to anisometropia, strabismus, or strabismic anisometropia were treated by primary occlusion. After termination of the occlusion therapy 85 patients $(47 \%)$ maintained their visual acuity, while in 97 patients $(53 \%)$ the visual acuity deteriorated and 'maintenance occlusion' had to be reinstituted. On the basis of the short term visual acuity results, the authors did not find any significant difference between the two groups with respect to the type of amblyopia.

The results of the present study indicate that low initial visual acuity and strabismic anisometropic amblyopia may significantly affect the long term of visual acuity results in eyes that earlier were successfully treated for amblyopia. We suggest that patients with these risk factors should be closely monitored for visual acuity up to the age of 9 years and repeatedly treated by patching whenever visual acuity shows deterioration.

1 Levartovsky S, Gottesman N, Shimshoni M, Oliver M. Factors affecting long-term results of successfully treated amblyopia. 7 Pediatr Ophthalmol Strabismus 1992; 29: 219-23.

2 Sparrow JC, Flynn JT. Amblyopia: a long-term follow-up. 7 Pediatr Ophthalmol 1977; 141: 333-6.

3 Gregersen E, Rindziunski OT. Conventional occlusion in the treatment of squint amblyopia. A 10-year follow-up Acta Ophthalmol 1965; 43: 462-74.

4 Malik SRK, Virdi PS, Goel BK. Follow-up results of occlusion and pleoptic treatment. Acta Ophthalmol 1975 53: $620-5$.

5 Aichmair H, Frey RG. Late results in the treatment of amblyopia. Klin Monatsbl Augenheilkd 1968; 153: 214-8.

6 Schropfer HD, Meinert K. Late results in amblyopia treatment. Klin Monatsbl Augenheilkd 1972; 166: 315-20.

7 Leydecker W, Ricklefs G, Ruhling R. Late results of amblyopia treatment. Klin Monatsbl Augenheilkd 1967; 151: 373-6.

8 Flynn JT, Cassady JC. Current trends in amblyopia therapy. Trans Am Acad Ophthalmol Otolaryngol 1978; 85: 428-50.

9 Ching FC, Parks MM, Friendly DS. Practical management of amblyopia. $\mathcal{f}$ Pediatr Ophthalmol Strabismus 1986; 23: 12-6.

10 Scott WE, Flabetich Dickey C. Stability of visual acuity in amblyopic patients after visual maturity. Graefes Arch Clin Exp Ophthalmol 1988; 226: 154-7.

11 Kutschke PJ, Scott WE, Keech RV. Anisometropic amblyopia. Ophthalmology 1991; 98: 258-63.

12 Oster JG, Simon JW, Jenkins P. When is it safe to stop patching? Br $₹$ Ophthalmol 1990; 74: 709-11. 